\title{
Intraoperative frozen section histological analysis of resection samples is useful for the control of primary lesions in patients with oral squamous cell carcinoma
}

\author{
AKIHIKO MIYAWAKI ${ }^{1}$, HIROSHI HIJIOKA ${ }^{2}$, TAKAYUKI ISHIDA ${ }^{2}$, ETSURO NOZOE $^{2}$, \\ NORIFUMI NAKAMURA ${ }^{2}$ and RYOICHI OYA ${ }^{1}$ \\ ${ }^{1}$ Department of Oral and Maxillofacial Surgery, University Hospital of Occupational and Environmental Health, Kitakyushu, \\ Fukuoka 807-8556; ${ }^{2}$ Department of Oral and Maxillofacial Surgery, Field of Oral and Maxillofacial Rehabilitation, \\ Advanced Therapeutics Course, Graduate School of Medical and Dental Sciences, \\ Kagoshima University, Kagoshima, Kagoshima 890-8520, Japan
}

Received July 9, 2014; Accepted August 22, 2014

DOI: $10.3892 / \mathrm{mco} .2014 .409$

\begin{abstract}
To ensure reliable surgical margins, intraoperative frozen section histological analysis (FS) has been performed since October, 2005 as follows: i) the orientation at the anatomical position and extent of the tumor are shared between oral pathologists and oral surgeons using imaging evaluations and pathological pictures and the planned site of sampling for intraoperative FS is confirmed; ii) a tumor team is organized and the team marks the tumor area and sets the resection range to correct the setting errors of the resection range among operators; iii) vital Lugol staining is applied to the lesion prior to tumor resection, the surgical margin is set based on the non-stained region and the extent of the tumor is macroscopically confirmed in the maximum cross-sectional surface of the resected specimen; and iv) FS is performed using samples from resected specimens to confirm the mucoepithelium and safety margin of the deep stump. The aim of this study was to evaluate the usefulness of our FS method. The treatment outcomes of oral squamous cell carcinoma were retrospectively investigated in patients treated prior to (Group 1) and after (Group 2) the introduction of our FS method. The recurrence rate of the primary lesions was high (17.3\%) in Group 1, but decreased significantly in Group 2 $(6.9 \%)$. Regarding clinicopathological factors, the condition of the surgical margins was associated with recurrence of the primary lesion in Group 1, but not in Group 2. In conclusion,
\end{abstract}

Correspondence to: Dr Akihiko Miyawaki, Department of Oral and Maxillofacial Surgery, University Hospital of Occupational and Environmental Health, 1-1 Iseigaoka Yahatanishi-ku Kitakyushu, Fukuoka 807-8556, Japan

E-mail: makihiko@clnc.uoeh-u.ac.jp

Key words: oral squamous cell carcinoma, control of primary lesion, surgical margins, locoregional recurrence, intraoperative frozen section histological analysis our FS method appears to be useful for resecting tumors with reliable safety margins.

\section{Introduction}

Locoregional control and treatment outcomes for primary oral cancers and cervical lymph node metastases have improved markedly with improvements in imaging diagnosis, advances in multidisciplinary treatment applying surgical therapy, radiotherapy and chemotherapy and the development of supportive therapies for oral cancer treatment (1-3). However, despite these advances, the primary lesion recurs in several cases. Therefore, control of the primary lesion is a major concern for oral surgeons, as recurrent lesions are difficult to control and markedly compromise the quality of life of the patients. In surgical therapy for oral cancers, the resection range for the primary lesion is determined based on the TNM classification following evaluation of the clinical findings and images from contrast-enhanced computed tomography (CT), contrast-enhanced magnetic resonance imaging (MRI), positron emission tomography-CT and ultrasonography (1). The safety margins of the resected primary lesion are confirmed during surgery by palpation and from intraoperative frozen section histological analysis (FS). However, the resection range varies among operators, the usefulness of FS has not been verified and the primary lesion recurs in several cases. As regards the methods used for evaluating the safety margins of the resected primary lesions, the 2013 guidelines for the treatment of oral cancer (1) described vital Lugol staining as being useful for mucosal lesions in cancer of the tongue. The recurrence rate of the primary lesions was found to be lower among patients for whom the non-Lugol-stained region was included in the resection field compared to those for whom there was no vital Lugol staining in the resected lesions. Although the examination of all the surgical margins of the resected primary lesions in FS is difficult and the scope of evaluation is limited, investigating the presence or absence of residual tumor tissue in the resected margin appears to be useful. Although actual methods for FS are not frequently 
reported, a survey of the American Head and Neck Society by Meier et al (4) stated that $76 \%$ of their members collected samples for FS from the surgical bed, 14\% from the resected specimens and the remaining $10 \%$ from both sites. There were no differences in the findings of FS regardless of the sampling site. Black et al (5) reported the actual condition of FS from the viewpoint of the pathologists, stating that the evaluation of the margins was inaccurate, as the anatomical orientation was not labeled in the resected specimens submitted to pathologists, which requires cooperation with the surgeons. Another report stated that FS is inappropriate for routine investigation of the margins for resected oral cancers other than tongue cancer, as the anatomical structure is complicated and anatomical limits mean that surgical access to the tumor site is generally poor (6). However, Wang et al (7) histopathologically examined the surgical margins of resected tumor specimens in FS using samples obtained by excisional biopsy and reported that no patient required additional treatment following surgery. Kurita et al (8) observed cross-sectional preparations of resected tumor specimens under a digital light microscope and reported that evaluation of the deep margin of the tumor was useful. Therefore, although FS was reported to be useful, there is yet no established method. To achieve accurate FS, it is important to share patient information with the pathologists, indicate the anatomical orientation of the resected tumor specimens and prepare samples from appropriate sites $(9,10)$. The advantages of FS using samples collected from resected tumor specimens are as follows: The anatomical orientation is readily determined; the distance between the surgical margin and tumor is macroscopically observed in the cross-sectional surface of the resected specimen; reliable sampling from an appropriate region is possible, as the anatomical orientation is readily determined; and the anatomical position of additional tumor resection is accurately reflected in the surgical field when the surgical margin is either close to the tumor or positive $(9,10)$. Based on these advantages, we collected samples from resected tumor specimens for FS.

To evaluate the usefulness of our FS system in the control of primary lesions, using methods such as intraoperative vital Lugol staining and FS of surgical specimens, the outcomes of treatment for oral squamous cell carcinoma (OSCC) were retrospectively investigated in patients treated prior to and after the introduction of this FS method to Kagoshima University.

\section{Materials and methods}

Patient eligibility criteria. The subjects comprised 153 patients with OSCC who underwent radical surgery at the Department of Oral and Maxillofacial Surgery at Kagoshima University between January, 2000 and September, 2011. The patients were divided according to whether they underwent surgery prior to or after adopting FS for the control of primary lesions in October, 2005 as follows: Group 1 (52 patients), treated between January, 2001 and September, 2005; and Group 2 (101 patients), treated from October, 2005 onwards. The preservation of the morphological characteristics of the oral cavity and functions such as mastication, swallowing, speech and esthetics is crucial in the treatment of advanced OSCC (11). Several studies have reported the effect of preoperative chemoradiotherapy plus radical surgery for advanced squamous cell carcinoma of the oral cavity (11-14). As a result, surgery was performed as the main treatment and chemoradiotherapy was performed as preoperative treatment throughout this period. Surgery comprised en bloc resection of the primary site, with neck dissection in N1 or more advanced cases. Chemoradiotherapy included external beam radiotherapy with a total radiation dose of 30-40 Gy delivered in 10-20 fractions and concurrent chemotherapy using either platinum-containing agents, such as cisplatin or carboplatin, 5-fluorouracil, or oral S-1. The clinical characteristics of the patients are summarized in Table I. There were no significant differences according to gender, age, primary site, or distribution of $\mathrm{T}$ or stage classification between the groups. However, more patients were treated with surgery alone in Group 2 compared to Group 1, as Group 1 included a higher number of advanced cases. The duration of the follow-up ranged from 1 year to 10 years and 8 months (median, 2 years and 8 months).

This study was approved by the Ethics Committee of Kagoshima University and written informed consent was obtained from all the included patients.

$F S$. To ensure reliable surgical margins, we have been performing FS for the control of primary lesions since October, 2005 as follows: First, the orientation of the anatomical extent is determined by oral pathologists and oral surgeons based on images obtained by contrast-enhanced CT and MRI and pathological pictures and the planned sampling site for FS is confirmed. Second, a tumor team is organized and marks the tumor area, setting a reliable $1-\mathrm{cm}$ resection range from the mark to correct the setting errors of the resection range by the operators. Third, only the presence or absence of tumor in tissues collected from the surgical bed of the tumor resection site is investigated in FS, but vital Lugol staining is applied (Fig. 1A) and the surgical margin is set based on the non-stained region. The distance from the tumor is macroscopically confirmed in the maximum cross-sectional surface of the resected specimen by oral surgeons and pathologists (Fig. 1B and C, white arrows). Finally, FS is performed using a sample collected from the resected specimen to confirm the mucoepithelium and safety margin of the deep stump (Fig. 1D).

Items analyzed in the two groups. First, the rates of positive surgical margins, recurrence of the primary lesion and disease-specific survival were compared. Second, the clinicopathological factors associated with recurrence of primary lesions were analyzed. The investigated clinicopathological factors included age, gender, tumor location, T classification, tumor properties, grade of differentiation, invasion pattern, presence or absence of lymphatic, vascular, or nerve invasion, condition of the surgical margins and histological therapeutic effect. The patients were divided by age into those aged $\geq 61$ and those $<60$ years, by $\mathrm{T}$ classification into $\mathrm{T} 2$ or lower and $\mathrm{T} 3$ or more advanced cases, by grade of differentiation into moderately or poorly differentiated and well-differentiated cases and by condition of the surgical margins into cases with residual tumor (positive margins), without residual tumor but $\leq 3 \mathrm{~mm}$ from the tumor, or without residual tumor and $>3 \mathrm{~mm}$ from the tumor (negative margins). The invasion pattern was classified as $\mathrm{YK} 3$ or lower and $\mathrm{YK} 4 \mathrm{C}$ or more advanced, according to 
Table I. Clinical characteristics of patients.

\begin{tabular}{|c|c|c|c|}
\hline Characteristics & $\begin{array}{l}\text { Group } 1, \text { no. }(\%) \\
(n=52)\end{array}$ & $\begin{array}{l}\text { Group } 2, \text { no. }(\%) \\
\qquad(\mathrm{n}=101)\end{array}$ & $\begin{array}{l}\text { Total patient no. }(\%) \\
\qquad(\mathrm{n}=153)\end{array}$ \\
\hline \multicolumn{4}{|l|}{ Gender } \\
\hline Male & $32(38.5)$ & $60(40.6)$ & $92(60.1)$ \\
\hline Female & $20(61.5)$ & $41(59.4)$ & $61(39.9)$ \\
\hline \multicolumn{4}{|l|}{ Age (years) } \\
\hline$<60$ & $13(25.0)$ & $33(32.7)$ & $46(30.0)$ \\
\hline$\geq 61$ & $39(75.0)$ & $68(67.3)$ & $107(70.0)$ \\
\hline \multicolumn{4}{|l|}{ Primary site } \\
\hline Upper gingiva & $6(11.5)$ & $10(9.9)$ & $16(10.5)$ \\
\hline Tongue & $23(44.2)$ & $52(51.5)$ & $75(49.0)$ \\
\hline Lower gingiva & $16(30.8)$ & $30(29.7)$ & $46(30.0)$ \\
\hline Other & $7(13.5)$ & $9(8.9)$ & $16(10.5)$ \\
\hline \multicolumn{4}{|c|}{ Clinical T classification } \\
\hline $\mathrm{T} 1 / 2$ & $37(71.2)$ & $83(82.2)$ & $120(78.4)$ \\
\hline $\mathrm{T} 3 / 4$ & $15(28.8)$ & $18(17.8)$ & $33(21.6)$ \\
\hline \multicolumn{4}{|l|}{ Stage } \\
\hline $\mathrm{I}$ & $10(19.2)$ & $18(17.8)$ & $28(18.3)$ \\
\hline II & $12(23.1)$ & $40(39.6)$ & $52(34.0)$ \\
\hline III & $19(36.5)$ & $24(23.8)$ & $43(28.1)$ \\
\hline IV & $11(21.2)$ & $19(18.8)$ & $30(19.6)$ \\
\hline \multicolumn{4}{|l|}{ Treatment } \\
\hline $\mathrm{S}$ & $8(15.4)$ & $54(53.4)$ & $62(40.5)$ \\
\hline $\mathrm{R} \rightarrow \mathrm{S}$ & $21(40.4)$ & $5(5.0)$ & $26(17.0)$ \\
\hline $\mathrm{R}+\mathrm{C} \rightarrow \mathrm{S}$ & $23(44.2)$ & $42(41.6)$ & $65(42.5)$ \\
\hline
\end{tabular}

$\mathrm{S}$, surgery; R, radiotherapy; C, chemotherapy.

the classification reported by Yamamoto et al (15). As regards the histological therapeutic effect, recurrence of the primary lesion was evaluated in patients who received preoperative therapy by dividing them into cases with Gr2a or lower and $\mathrm{Gr} 2 \mathrm{~b}$ or higher effects, according to the classification reported by Shimosato et al (16). Third, disease-specific survival rates were compared between the groups according to the condition of the surgical margins. Finally, the primary site, condition of the surgical margin, time of recurrence and prognosis were analyzed in cases with recurrence of the primary lesion in Groups 1 and 2.

Statistical analysis. Statistical analysis was performed using $\mathrm{JMP}^{\circledR}$ statistical analysis software, version 9 (SAS Institute, Tokyo, Japan). The associations between recurrence rate and clinicopathological factors were analyzed using the Pearson's $\chi^{2}$ test. The survival rates were calculated using the Kaplan-Meier method and analyzed using the log-rank test. $\mathrm{P}<0.05$ was considered to indicate a statistically significant difference.

\section{Results}

Comparison of surgical margin positivity, primary lesion recurrence and disease-specific survival by the Kaplan-Meier method. The surgical margin positivity rates were 9.6 and $3.9 \%$ in Groups 1 and 2, respectively, with a decreasing tendency, although the difference was not statistically significant (Table II). The recurrence rate for primary lesions was high $(17.3 \%, 9 / 52)$ in Group 1, but improved significantly to $6.9 \%$ (7/101) in Group 2 (Table II). Disease-specific survival rates were 81.5 and $87.9 \%$ in Groups 1 and 2, respectively, showing a slight but non-significant tendency toward improvement (Fig. 2).

Clinicopathological factors associated with recurrence of the primary lesions. The Pearson's $\chi^{2}$ test was performed regarding the presence or absence of recurrence of the primary lesion as a response variable and gender, age, location, T classification, tumor properties, grade of differentiation, invasion pattern, presence or absence of lymphatic, vascular, or nerve invasions, condition of the surgical margins and histological therapeutic effect as explanatory variables. In Group 1, factors associated with recurrence of the primary lesion were the presence or absence of nerve invasion and the condition of the surgical margins; recurrence rate was found to be significantly higher among cases with surgical margins close to the tumor or residual tumor in the surgical margins (positive margins). In Group 2, none of the explanatory factors were significantly associated with the presence or absence of recurrence of the primary lesion. Regarding the association between primary 


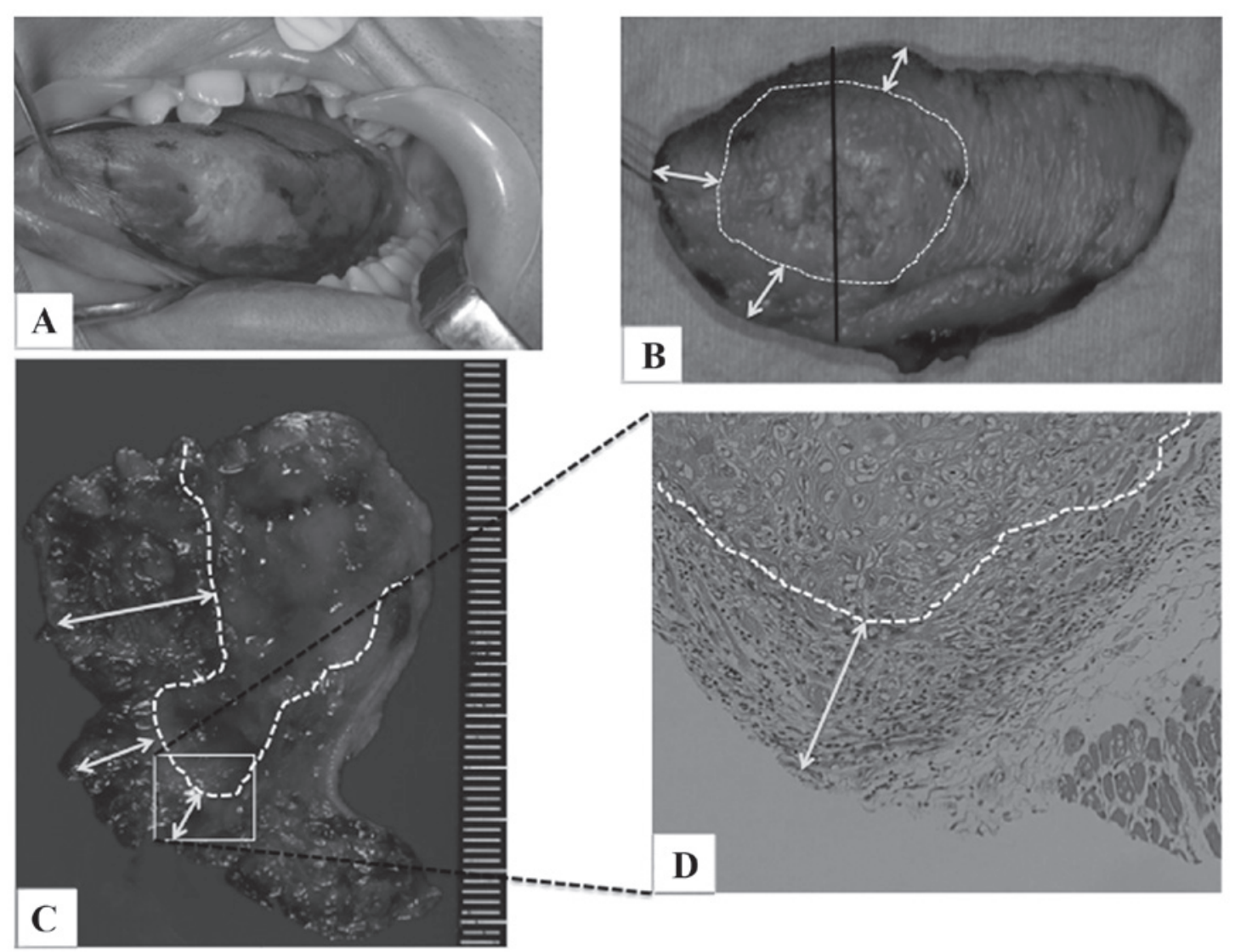

Figure 1. Intraoperative frozen section histological analysis method. (A) A case of T1 cancer of the tongue. Vital Lugol staining was applied during surgery and the surgical margins $10 \mathrm{~mm}$ from the tumor were determined. (B) Resected tumor specimen. The specimen was cut in cross-section (black line) in the center of the tumor (region circled with white dotted line) with a palpable induration. The white arrows show the distance between the surgical margins and the tumor macroscopically. (C) The cross-sectional surface of the tumor was observed macroscopically to evaluate the surgical margins (the white dotted line represents the tumor margin). The white arrows show the distance between the surgical margins and the tumor. (D) Hematoxylin and eosin staining (magnification, x200). A sample was collected from the deepest region close to the macroscopic tumor and subjected to intraoperative rapid pathological examination. The white arrows shows the distance between the region demarcated by the white dotted line and the surgical margins microscopically.

Table II. Rates of negative surgical margins and recurrence at primary site in Groups 1 and 2.

\begin{tabular}{lccc}
\hline Variables & Group 1 & Group 2 & P-value \\
\hline Margins & & & \\
Positive & 47 & 97 & \\
Negative (\%) & $5(9.6)$ & $4(3.9)$ & 0.16 \\
Recurrence & & & \\
No & 43 & 94 & \\
Yes (\%) & $9(17.3)$ & $7(6.9)$ & $0.047^{\text {a }}$ \\
\hline
\end{tabular}

${ }^{\text {ap }}<0.05$ (Pearson's $\chi^{2}$ test).

site and recurrence of the primary lesion, primary lesions in the upper and lower gingiva frequently recurred in both groups, but the incidence decreased in Group 2 and cancer of the tongue recurred in only 1 patient (Table III).

Disease-specific survival rate by condition of the surgical margins in Groups 1 and 2. In Group 1, the survival rate was $87.8 \%$ in cases with negative surgical margins, $72.8 \%$ in cases with margins close to the tumor and $60.0 \%$ in cases with

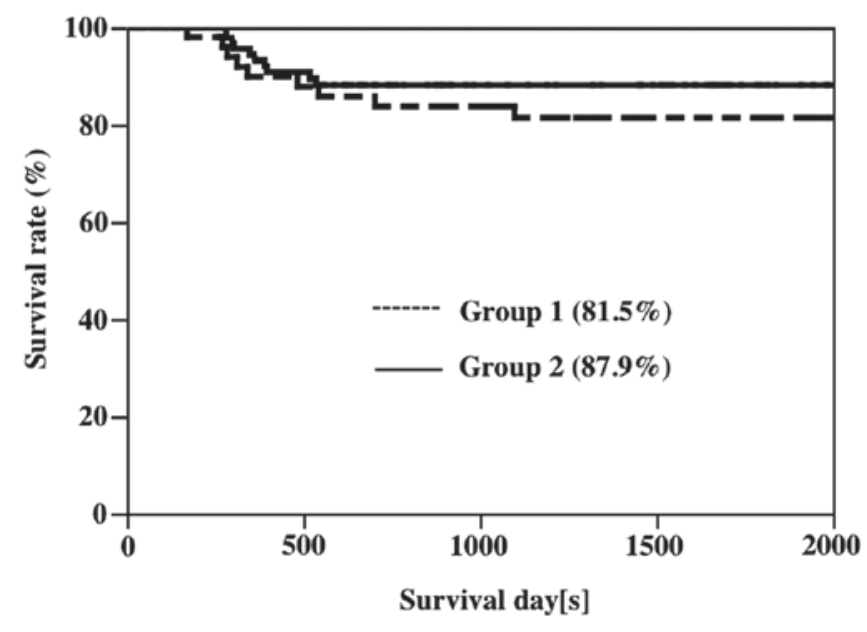

Figure 2. Disease-specific survival rates in Groups 1 and 2.

positive margins. In Group 2, the survival rates of cases with negative margins and cases with margins close to the tumor were 93.3 and $78.3 \%$, respectively, exhibiting a tendency toward higher rates compared to those in Group 1, although the differences were not significant. The disease-specific survival rate in positive-margin cases was $50.0 \%$, which was 
Table III. Clinicopathological factors associated with recurrence at primary site.

\begin{tabular}{|c|c|c|c|c|c|c|}
\hline \multirow[b]{2}{*}{ Variables } & \multicolumn{3}{|c|}{ Group 1} & \multicolumn{3}{|c|}{ Group 2} \\
\hline & No recurrence & $\begin{array}{c}\text { Recurrence } \\
\text { no. }(\%)\end{array}$ & P-value & No recurrence & $\begin{array}{c}\text { Recurrence } \\
\text { no. }(\%)\end{array}$ & $\mathrm{P}$-value \\
\hline \multicolumn{7}{|l|}{ Gender } \\
\hline Male & 28 & 4 & & 57 & 4 & \\
\hline Female & 15 & 5 & 0.25 & 37 & 3 & 0.36 \\
\hline \multicolumn{7}{|l|}{ Age (years) } \\
\hline$\geq 61$ & 12 & 1 & & 32 & 1 & \\
\hline$<60$ & 31 & 8 & 0.29 & 62 & 6 & 0.28 \\
\hline \multicolumn{7}{|l|}{ Primary site } \\
\hline Upper gingiva & 4 & $2(33.3)$ & & 8 & $2(20.0)$ & \\
\hline Tongue & 20 & $3(13.0)$ & & 51 & $1(1.9)$ & \\
\hline Lower gingiva & 13 & $3(18.8)$ & & 26 & $4(13.3)$ & \\
\hline Other & 6 & 1 & 0.56 & 9 & 0 & 0.12 \\
\hline \multicolumn{7}{|l|}{ Clinical T classification } \\
\hline $\mathrm{T} 1 / 2$ & 32 & 5 & & 78 & 5 & \\
\hline $\mathrm{T} 3 / 4$ & 11 & 4 & 0.26 & 16 & 2 & 0.44 \\
\hline \multicolumn{7}{|l|}{ Pattern of tumor growth } \\
\hline Superficial spreading & 6 & 0 & & 22 & 3 & \\
\hline Outgrowing & 2 & 0 & & 24 & 1 & \\
\hline Ingrowing & 35 & 9 & 0.37 & 48 & 3 & 0.49 \\
\hline \multicolumn{7}{|l|}{ Differentiation } \\
\hline Moderate/poor & 29 & 7 & & 81 & 7 & \\
\hline High & 14 & 2 & 0.54 & 13 & 0 & 0.29 \\
\hline \multicolumn{7}{|l|}{ Mode of invasion ${ }^{\mathrm{b}}$} \\
\hline$\leq \mathrm{YK} 3$ & 36 & 6 & & 76 & 4 & \\
\hline YK4C/4D & 7 & 3 & 0.24 & 18 & 3 & 0.16 \\
\hline \multicolumn{7}{|l|}{ Lymphatic invasion } \\
\hline Negative & 39 & 7 & & 82 & 6 & \\
\hline Positive & 4 & 2 & 0.27 & 11 & 1 & 0.85 \\
\hline \multicolumn{7}{|l|}{ Vascular invasion } \\
\hline Negative & 37 & 7 & & 76 & 6 & \\
\hline Positive & 6 & 2 & 0.53 & 17 & 1 & 0.79 \\
\hline \multicolumn{7}{|l|}{ Nerve invasion } \\
\hline Negative & 42 & 7 & & 86 & 7 & \\
\hline Positive & 1 & 2 & $0.02^{\mathrm{a}}$ & 7 & 0 & 0.45 \\
\hline \multicolumn{7}{|l|}{ Surgical margin } \\
\hline Negative & 32 & 3 & & 73 & 4 & \\
\hline Close $(<3 \mathrm{~mm})$ & 9 & 3 & & 17 & 3 & \\
\hline Positive & 2 & 3 & $0.01^{\mathrm{a}}$ & 4 & 0 & 0.21 \\
\hline \multicolumn{7}{|l|}{ Chemoradiation effect $^{\mathrm{c}}$} \\
\hline$\leq \mathrm{Gr} 2 \mathrm{a}$ & 11 & 5 & & 12 & 2 & \\
\hline$\geq \mathrm{Gr} 2 \mathrm{~b}$ & 22 & 3 & 0.13 & 30 & 1 & 0.17 \\
\hline
\end{tabular}

${ }^{\mathrm{a}} \mathrm{P}<0.05$ (Pearson's $\chi^{2}$ test). ${ }^{\mathrm{b}} \mathrm{Classification}$ reported by Yamamoto et al (15). ${ }^{\mathrm{c} C l a s s i f i c a t i o n}$ reported by Shimosato et al (16).

lower compared to that in Group 1. Significant differences according to the condition of the surgical margins were noted in the survival rates of both groups (Fig. 3).
Patients with recurrence of primary lesions in Groups 1 and 2 and outcome. In Group 1, the primary tumors recurred in 9 of the 52 patients $(17.3 \%)$. By primary site, recurrence 
Table IV. Cases of recurrence at primary site and prognosis.

\begin{tabular}{|c|c|c|c|c|c|c|c|c|c|}
\hline Case & $\begin{array}{c}\text { Age } \\
\text { (years) }\end{array}$ & Gender & $\begin{array}{l}\text { Primary } \\
\text { site }\end{array}$ & $\begin{array}{c}\mathrm{TN} \\
\text { stage }\end{array}$ & $\begin{array}{l}\text { Surgical } \\
\text { margins }\end{array}$ & $\begin{array}{l}\text { Site of } \\
\text { recurrence }\end{array}$ & $\begin{array}{l}\text { Time to } \\
\text { recurrence }\end{array}$ & $\begin{array}{l}\text { Salvage } \\
\text { treatment }\end{array}$ & Outcome \\
\hline \multicolumn{10}{|c|}{ Group 1} \\
\hline 1 & 52 & Female & Upper gingiva & $\mathrm{T} 2 \mathrm{~N} 1$ & Close & Skin & $3 y 2 \mathrm{~m}$ & Excision & Alive \\
\hline 2 & 63 & Male & Upper gingiva & T3N0 & Close & Buccal mucosa & $5 \mathrm{~m}$ & Excision & Alive \\
\hline 3 & 70 & Female & Tongue & T1N0 & Negative & Tongue & $3 y 11 \mathrm{~m}$ & Excision & Alive \\
\hline 4 & 67 & Male & Tongue & T2N0 & Negative & Tongue & 1 y $5 \mathrm{~m}$ & Excision & Deceased \\
\hline 5 & 71 & Male & Tongue & $\mathrm{T} 2 \mathrm{~N} 1$ & Negative & Skin & $3 y$ & Excision & Alive \\
\hline 6 & 62 & Male & Lower gingiva & $\mathrm{T} 4 \mathrm{~N} 2 \mathrm{~b}$ & Positive & Retromolar & $3 \mathrm{~m}$ & Chemotherapy & Alive \\
\hline 7 & 68 & Female & Lower gingiva & T2N0 & Positive & Skin & $1 \mathrm{~m}$ & - & Deceased \\
\hline 8 & 86 & Female & Lower gingiva & T2N1 & Close & Gingiva & $5 \mathrm{~m}$ & Chemotherapy & Deceased \\
\hline 9 & 84 & Female & Buccal mucosa & T3N0 & Positive & Buccal mucosa & $1 \mathrm{~m}$ & Excision & Deceased \\
\hline \multicolumn{10}{|c|}{ Group 2} \\
\hline 10 & 66 & Male & Upper gingiva & $\mathrm{T} 2 \mathrm{~N} 2 \mathrm{~b}$ & Negative & Buccal mucosa & $1 y$ & Radiotherapy & Alive \\
\hline 11 & 84 & Female & Upper gingiva & T3N0 & Close & Skin & $7 \mathrm{~m}$ & Excision & Deceased \\
\hline 12 & 81 & Female & Tongue & T4N0 & Negative & Tongue & $1 \mathrm{y}$ & Radiotherapy & Deceased \\
\hline 13 & 72 & Male & Lower gingiva & T4N1 & Close & Skin & $4 \mathrm{~m}$ & Excision & Deceased \\
\hline 14 & 81 & Female & Lower gingiva & T2NO & Negative & Skin & $3 \mathrm{~m}$ & Excision & Alive \\
\hline 15 & 84 & Female & Lower gingiva & T4N0 & Close & Gingiva & $5 \mathrm{~m}$ & - & Deceased \\
\hline 16 & 60 & Female & Lower gingiva & T2N0 & Negative & Gingiva & $1 \mathrm{y} 9 \mathrm{~m}$ & Excision & Alive \\
\hline
\end{tabular}

Y, years; m, months.
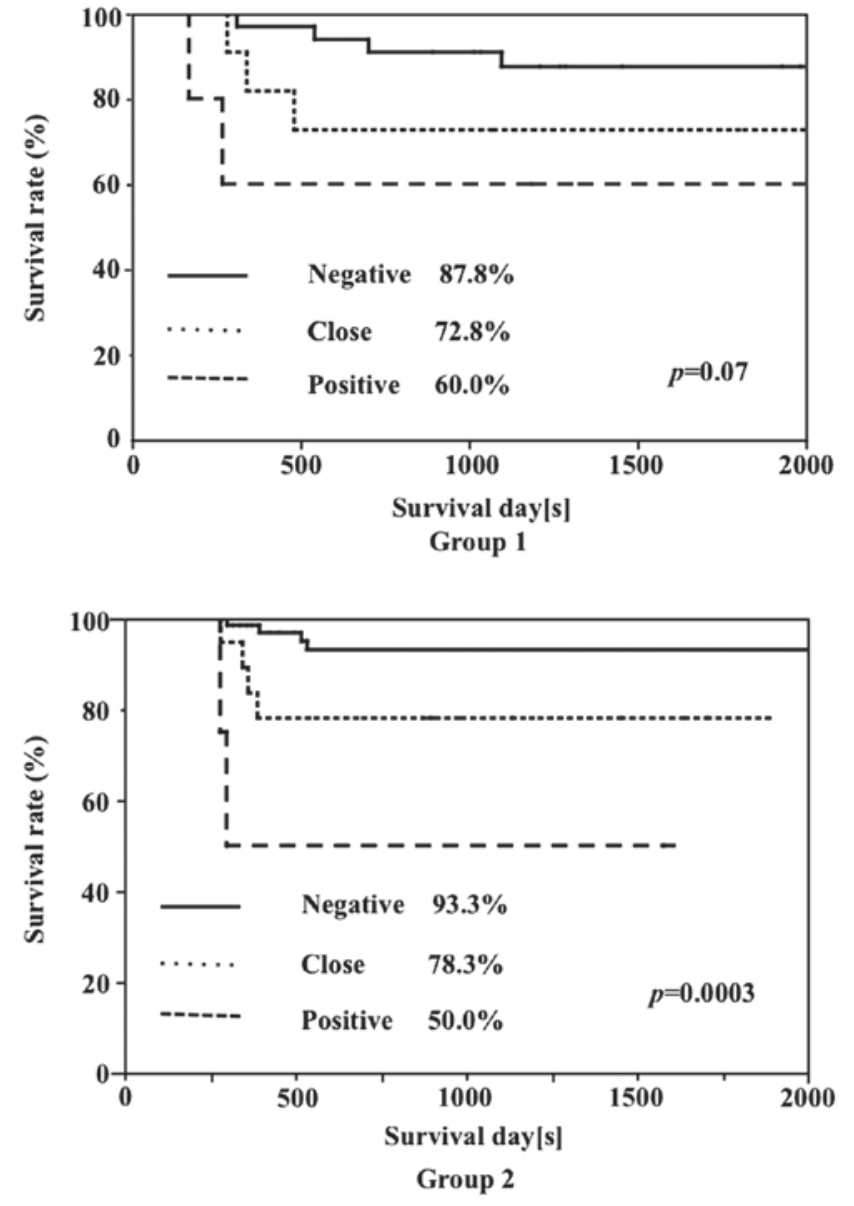

Figure 3. Disease-specific survival rate according to the surgical margin in Groups 1 and 2. occurred in the upper gingiva in 2 patients, tongue in 3, lower gingiva in 3 and buccal mucosa in 1 patient. The $\mathrm{T}$ classification varied between $\mathrm{T} 1$ and $\mathrm{T} 4$ and the surgical margins were negative, close to the tumor and positive in 3 patients each. The recurrence site was the tongue, gingiva, buccal mucosa and retromolar mucosa around the primary site in 6 patients and the tumor advanced into the skin and recurred in 3 patients. The time to recurrence was between 1 and 3 months in cases with positive margins, after 5 months in 2 cases with close margins and significantly later in cases with negative margins (range, 1 year and 5 months to 3 years and 11 months).

The treatment comprised tumor resection or chemotherapy in 8 patients and 5 patients $(62.5 \%)$ survived, but the outcomes were poor and 4 patients $(37.5 \%)$ succumbed to the primary tumor.

In Group 2, the primary lesions recurred in 7 of the 101 patients $(6.9 \%)$. The primary site was located in the upper and lower gingiva in 6 cases and in the tongue in 1 case. The $\mathrm{T}$ classification was late $\mathrm{T} 2$ or more advanced and the surgical margins were negative in 4 and close to the tumor in 3 cases; however, no positive cases were recorded. The site of recurrence was the tongue, gingiva and buccal mucosa around the primary lesion in 4 patients and the skin in 3 patients. The time to tumor recurrence was 4-7 months in cases with close margins, $>1$ year in 2 cases with negative margins, but only 3 months after surgery in 1 case with negative margins. The treatment comprised radiotherapy or resection in 6 patients, of whom $3(50 \%)$ survived and 3 succumbed to the primary lesion. One patient with lower gingival cancer was untreatable and eventually succumbed to the disease. The characteristics of the cases with recurrence of the primary tumor are summarized in Table IV. 


\section{Discussion}

The major clinical factor determining the prognosis of patients with OSCC is cervical lymph node metastasis, whereas the depth and pattern of invasion are important factors associated with recurrence of the primary lesion and lymph node metastasis (1). In addition to the depth and invasion pattern of the tumor, the presence or absence of tumor cells in the surgical margins is crucial for the surgical treatment of OSCC $(17,18)$. Setting a safety margin $\geq 10 \mathrm{~mm}$ is considered as appropriate for the resection of oral cancers, although a clear basis for this distance is currently lacking (19). We have attempted to control primary lesions by following this criterion (10-mm safety margin), confirming that the region remains unstained on vital Lugol staining during surgery and including this region in the resection field, confirming the macroscopic tumor extent in the cross-sectional surface of the resected specimen and performing FS for a sample collected from the resected specimen. Although the disease-specific survival rate was not significantly affected, the rate of positive surgical margins was decreased. The rate of primary lesion recurrence was high $(17.3 \%, 9 / 52)$ in Group 1, but improved significantly to $6.9 \%$ (7/101) in Group 2 . Among the clinicopathological factors, the condition of the surgical margins and the presence or absence of nerve invasion were associated with recurrence of the primary lesion in Group 1, but no significant association between the surgical margin status and recurrence of the primary lesion was observed in Group 2. However, the prognosis of patients with positive margins was poor in both groups and, although the incidence of recurrent cancer of the tongue tended to decrease, upper and lower gingival cancers recurred in a number of patients, reflecting the limitations to our approach for the control of primary lesions.

The number of studies reporting the recurrence rate of primary lesions in detail is limited. Although the rates vary depending on the primary site, Yamamoto et al (18) reported a rate of $10.3 \%$ in patients with T1/2 cancer of the tongue, whereas that of oral cancers of other regions, including the tongue, was reported to be $9-18 \%$ by other studies $(18,20-22)$. Although a simple comparison with these reports is not feasible due to the differences in patient background and treatment strategy, the rate of primary lesion recurrence was $17.3 \%$ in Group 1, which was similar to the previously reported rates, and decreased to $6.9 \%$ in Group 2, which was lower compared to the rates reported elsewhere. In addition, among the clinicopathological factors, the condition of the surgical margins and nerve invasion were associated with recurrence of the primary lesion in Group 1, while no significant correlation was noted between surgical margin status and recurrence of the primary lesion in Group 2. Surgical margin positivity represents a significant factor associated with decreased survival rate and a high risk of postoperative recurrence $(1,22)$. The condition of the surgical margins was significantly associated with survival rate in both groups (Fig. 3), suggesting that our approach for the control of primary lesions contributes to decreasing the risk of recurrence and our FS method appears to be useful for the evaluation of the surgical margins. However, the survival rate did not significantly improve in Group 2 compared to that in Group 1, although a tendency towards an increase was observed. The poor prognosis of patients with cervical lymph node metastasis, including secondary cervical lymph node metastasis in Group 2 (data not shown), may have affected our results.

The recurrence rate of the primary lesions varies depending on the primary site. The oral cavity has a complex structure, comprising mixed hard and soft tissues and the invasion pattern varies depending on the direction of tumor advancement. Such factors may contribute to the difficulties in the determination of the resection range with adequate safety margins (1). Recurrence of the primary lesion was frequently noted in the upper and lower gingiva in both groups. This tendency persisted in Group 2, but the incidence was decreased in all the primary sites. As regards cancer of the tongue, a low rate of primary lesion recurrence (3.8\%) has been reported (15). In our patients with cancer of the tongue, the rate of primary site recurrence was $13.0 \%$ in Group 1, but decreased to $1.9 \%$ in Group 2. In Group 2, recurrence occurred in the upper and lower gingiva in 2 and 4 patients, respectively (Table IV), but recurrence in the tongue occurred in only 1 case. The advances in imaging diagnosis may also be a decisive factor when determining the resection range, but the advantages of our FS method (i.e., the cross-sectional surface of tumors is readily observed macroscopically, the distance between the surgical margin and tumor is readily determined and the anatomical orientation is readily identified) is evident in tissues retaining anatomical continuity, such as the tongue, which may facilitate determining a reliable resection range for cancer of the tongue. In Group 2, although recurrence was negative on intraoperative rapid pathological diagnosis, upper and lower gingival cancers recurred in the surrounding tissue relatively early after surgery (3-7 months) in 4 of the 6 patients. These cases reflect the limitations of our FS method in assisting with determining a reliable tumor resection range, in addition to the difficulties involved in imaging diagnosis of tumors located in regions with a complex anatomical structure, such as advanced upper and lower gingival cancers containing hard as well as soft tissues. The prognosis for cases with recurrence is very poor $(23,24)$. To determine the resection range for the primary lesion in such cases, further improvements are required in the imaging evaluation of jaw bone infiltration, tumor invasion pattern and infiltration into the surrounding soft tissues in consideration of the direction of tumor advancement (25).

In conclusion, our FS method appears to be useful for resecting tumors with reliable safety margins for tissues retaining anatomical continuity, such as the tongue. The macroscopic observation of cross-sections of the resected tumor specimens is easy and the surgical margins may be readily investigated. However, this method is insufficient for determining a resection range in tissues containing soft tissue and jaw bone, such as upper and lower gingival tumors, and other methods to control primary lesions must be investigated.

\section{Acknowledgements}

The authors would like to thank the members of the Department of Oral and Maxillofacial Surgery, Field of Oral and Maxillofacial Rehabilitation, Advanced Therapeutics Course, Graduate School of Medical and Dental Sciences, Kagoshima University, for their assistance with additional data collection. 


\section{References}

1. Joint Committee of Guidelines for Treatment of Oral Cancers Working Group of the Japan Society for Oral Tumors and Oral Cancer Clinical Practice Guidelines Development Committee of the Japan Society of Oral and Maxillofacial Surgeons. 2013 evidence-based oral cancer clinical practice guidelines. Kanahara \& Co., Tokyo, pp37-39, 65-87, 85, 91, 2013 (In Japanese)

2. Pfister DG, Ang KK, Brizel DM, et al; National Comprehensive Cancer Network: Head and neck cancers, version 2.2013. Featured update to the NCCN guidelines. J Natl Compr Canc Netw 11: 917-923, 2013.

3. Balasundaram I, AI-Hadad I and Parmar S: Recent advances in reconstructive oral and maxillofacial surgery. Br J Oral Maxillofac Surg 50: 695-705, 2012.

4. Meier JD, Oliver DA and Varvares MA: Surgical margin determination in head and neck oncology: current clinical practice. The results of an International American Head and Neck Society Member Survey. Head Neck 27: 952-958, 2005.

5. Black C, Marotti J, Zarovnaya E, et al: Critical evaluation of frozen section margins in head and neck cancer resections. Cancer 107: 2792-2800, 2006.

6. Gerber S, Gengler C, Gratz KW, et al: The impact of frozen sections on final surgical margins in squamous cell carcinoma of the oral cavity and lips: a retrospective analysis over an 11 years period. Head Neck Oncol 3: 56, 2011.

7. Wang YC, Fang KH, Jung SM, et al: Excisional biopsy with margin control for oral cancers. Head Neck 32: 1528-1533, 2010

8. Kurita H, Uehara S, Funamoto S, et al: Intraoperative digital microscopic assessment of the deep surgical margins in oral carcinoma survey: a preliminary report. Am J Surg 191: 84-88, 2006.

9. Gauthier P, Audet N, Guertin L, et al: Complete frozen section margins (with measurable 1 or $5 \mathrm{~mm}$ thick free margin) for cancer of the tongue: part 2: clinical experience. J Otolaryngol Head Neck Surg 39: 20-27, 2010.

10. Hinni ML, Zarka MA and Hoxworth JM: Margin mapping in transoral surgery for head and neck cancer. Laryngoscope 123: 1190-1198, 2013.

11. Miyawaki A, Ikeda R, Hijioka H, et al: SUVmax of FDG-PET correlates with the effects of neoadjuvant chemoradiotherapy for oral squamous cell carcinoma. Oncol Rep 23: 1205-1212, 2010.

12. Miyawaki A, Hijioka H, Ikeda R, et al: Analysis of the outcome of concurrent neoadjuvant chemoradiotherapy with S-1 compared to super-selective intra-arterial infusion for oral squamous cell carcinoma. Oncol Lett 3: 995-1001, 2012.

13. Kirita T, Ohgi K, Shimooka H, et al: Preoperative concurrent chemoradiotherapy plus radical surgery for advanced squamous cell carcinoma of the oral cavity: an analysis of long-term results. Oral Oncol 35: 597-606, 1999.
14. Mohr C, Bohndorf W, Carstens J, et al: Preoperative radiochemotherapy and radical surgery in comparison with radical surgery alone. A prospective, multicentric, randomized DOSAK study of advanced squamous cell carcinoma of the oral cavity and the oropharynx (a 3-year follow up). Int J Oral Maxillofac Surg 23: 140-148, 1994.

15. Yamamoto E, Kohama G, Sunakawa H, et al: Mode of invasion, bleomycine sensitivity, and clinical course in squamous cell carcinoma of the oral cavity. Cancer 51: 2175-2180, 1983.

16. Shimosato Y, Oboshi S and Baba K: Histological evaluation of effects of radiotherapy and chemotherapy for carcinoma. J Clin Oncol 1: 19-35, 1971.

17. Ling W, Mijiti A and Moming A: Survival pattern and prognostic factors of patients with squamous cell carcinoma of the tongue: a retrospective analysis of 210 cases. J Oral Maxillofac Surg 71: 775-785, 2013

18. Yamamoto S, Yamada S, Takahasi S, et al: Clinicopathological risk factors for local recurrence in oral squamous carcinoma. Int J Oral Maxillofac Surg 41: 1195-1200, 2012.

19. Nason RW, Binahmed A, Pathak KA, et al: What is the adequate margin of surgical resection in oral cancer? Oral Surg Oral Med Oral Pathol Oral Radiol Endod 107: 625-629, 2009.

20. Yanamoto S, Yamada S, Takahashi H, et al: Predictors of locoregional recurrence in T1-2N0 tongue cancer patients. Pathol Oncol Res 19: 795-803, 2013.

21. Po Wing Yuen A, Lam KY, Lam LK, et al: Prognostic factors of clinically stage I and II oral tongue carcinoma - A comparative study of stage, thickness, shape, growth pattern, invasive front malignancy grading, Martinez-Gimeno score, and pathologic features. Head Neck 24: 513-520, 2002.

22. Woolgar JA, Rogers S, West CR, et al: Survival and patterns of recurrence in 200 oral cancer patients treated by radical surgery and neck dissection. Oral Oncol 35: 257-265, 1999.

23. Jones AS, Bin Hanafi Z, Nadapalan V, et al: Do positive resection margins after ablative surgery for head and neck cancer adversely affect prognosis? A study of 352 patients with recurrent carcinoma following radiotherapy treated by salvage surgery. $\mathrm{Br}$ J Cancer 74: 128-132, 1996.

24. Kemohan MD, Clark JR, Gao K, et al: Predicting the prognosis of oral squamous cell carcinoma after first recurrence. Arch Otolaryngol Head Neck Surg 136: 1235-1239, 2010.

25. Feichtinger M, Pau M, Zemann W, et al: Intraoperative control of resection margins in advanced head and neck cancer using a 3D-navigation system based on PET/CT image fusion. J Craniomaxillofac Surg 38: 589-594, 2010. 